Original Article

\title{
ISOLATION, CHARACTERIZATION AND PREDICTION OF BIOLOGICAL ACTIVITY OF TWO NEW FATTY ESTERS AND A PHENOL FROM THE HEARTWOOD OF PTEROCARPUS MARSUPIUM ROXB.
}

\author{
DEEPTI KATIYAR ${ }^{*}$, VIJENDER SINGH ${ }^{2}$, MOHAMMED ALI ${ }^{3}$ \\ 1Department of Pharmacognosy, KIET School of Pharmacy, Ghaziabad, UP, India, ${ }^{2}$ School of Pharmacy, Sharda University, Greater Noida, \\ UP, India, ${ }^{3}$ Department of Pharmacognosy and Phytochemistry, Faculty of Pharmacy, Jamia Hamdard, New Delhi, India \\ Email: katiyar_deepti@yahoo.co.in
}

Received: 29 Oct 2016 Revised and Accepted: 07 Dec 2016

\begin{abstract}
Objective: The current investigation involves the isolation, characterization and prediction of biological activity spectra of the phytoconstituents from the ethanolic extract of the heartwood of Pterocarpus marsupium roxb. (Fabaceae).

Methods: The heartwood ( $3 \mathrm{~kg}$ ) was extracted in alcohol by cold maceration for $21 \mathrm{~d}$ and the compounds were isolated by column chromatography. The compounds thus isolated were characterised and their structures were elucidated by using assorted spectral data analysis, i.e., infrared radiation spectroscopy (IR), proton nuclear magnetic resonance $\left({ }^{1} \mathrm{HNMR}\right)$, carbon thirteen nuclear magnetic resonance $\left({ }^{13} \mathrm{C}\right.$ NMR) and direct analysis in real time mass spectrometry (DART-MS). PASS (prediction of activity spectra for substances) computer program was used to predict the biological activity spectra of the isolated compounds.
\end{abstract}

Results: Phytochemical investigation of ethanolic extract of the heartwood of Pterocarpus marsupium led to isolate two fatty esters and a phenolic compound characterised as $n$-octanyl $n$-octadeca-9,12-dienoate (n-octanyl linoleiate, 1 ), $n$-dodecanyl $n$-octadeca-9,12-dienoate (n-dodecanyl linoleiate, 2) and 2, 3-dioxymethylene phenol (3). These phytoconstituents are reported for first time in the heartwood of Pterocarpus marsupium Roxb. The in silico profiling of these phytoconstituents exhibited their broad spectra of biological activity. Compounds (1) and (2) showed their maximum activity as All-trans-retinyl-palmitate hydrolase inhibitor, anti eczematic, lipid metabolism regulator, etc. and compound (3) was found to be active as membrane integrity agonist, aspulvinone dimethylallyl transferases inhibitor, carminative, neurotransmitter uptake inhibitor, etc.

Conclusion: These isolated phytoconstituents can be used as the marker compounds to establish the identity, quality and purity of the drug. The results of PASS prediction shall be very useful for establishing these phytoconstituents as active pharmacological moieties.

Keywords: 2, 3-dioxymethylene phenol, Fabaceae, Fatty esters, Heartwood, Pterocarpus marsupium, PAAS

(C) 2017 The Authors. Published by Innovare Academic Sciences Pvt Ltd. This is an open access article under the CC BY license (http://creativecommons.org/licenses/by/4. 0/) DOI: http://dx.doi.org/10.22159/ijpps.2017v9i2.15938

\section{INTRODUCTION}

The wealthiest bio-resource of drugs includes the medicinal plants [1]. They have been extensively used by the traditional healers for treatment of various diseases [2]. One such traditional medicinal plant-Pterocarpus marsupium was selected for the current investigation

Pterocarpus marsupium Roxb. (Fabaceae), also known as Malabar kino, Indian kino tree or Vijayasar, is a medium to large, deciduous tree that can grow up to $30 \mathrm{~m}$ tall with compound and imparipinnate leaves, terminal panicles of yellow flowers, flat, circular and winged pod, convex and bony seeds and dark brown to grey bark with surface fissures. It is native to India, Nepal and Sri Lanka, where it occurs in parts of the Western Ghats in the Karnataka-Kerala region and also in the forests of central India $[3,4]$.

Its heartwood is used as an astringent and to treat inflammation, diabetes, obesity, diarrhea, vitiligo, eczema, psoriasis and bleeding [5-8]. The heartwood and other parts of the plant contained pterostilbene, pterocarpol, flavonoids, 1-(2', 6'-dihydroxyphenyl)- $\beta$ D-glucopyranoside, lupeol, phytosterols, p-hydroxybenzaldehyde and pterocarposide [9-16].

Every phytoconstituent exhibits a broad spectrum of effects. Some may be beneficial and used for the treatment of various diseases while others may be toxic. Modern drug design and discovery utilise the various web-services for the prediction of physicochemical properties, biological activity and toxicity of chemical compounds. PASS is one such computer program which is able to evaluate any new compound in huge chemical-pharmacological space $[17,18]$.
The main objective of our study was to isolate, characterise and predict the biological activity spectra for the phytoconstituents from the heartwood of Pterocarpus marsupium.

\section{MATERIALS AND METHODS}

General experimental procedures

All melting points (mp) were determined in centigrade scale in oneend open capillary on a thermoelectrical melting point apparatus. The IR spectra were measured on IR affinity-1 Fourier transform infrared spectrometer model (Schimadzu). The mass spectra were recorded on a JEOL-Accu TOF (time of flight) JMS-T100LC mass spectrometer having a DART (direct analysis in real time) source. The $\mathrm{m} / \mathrm{z}$ (mass to charge ratio) values of the more intense peaks are mentioned and the fig. in a bracket attached to each $\mathrm{m} / \mathrm{z}$ values indicated relative intensities with respect to the base peak. The ${ }^{1} \mathrm{H}$ and ${ }^{13} \mathrm{C}$ NMR spectra were scanned on Bruker AvIII HD-300 and 75 $\mathrm{MHz}$, respectively, an instrument in $\mathrm{CDCl}_{3}$ and $\mathrm{MeOD}$ solvents using TMS as an internal standard. The coupling constants (J values) are expressed in Hertz (Hz). Column chromatography was performed on a silica gel (60-120 mesh; Qualigen, Mumbai, India) column. TLC (thin layer chromatography) was run on silica gel G 60 F 254 (Qualigen) coated aluminium sheets. Spots were visualised by exposing to iodine vapors, UV (ultraviolet) radiation and spraying with ceric sulfate solution.

\section{Plant material}

The plant material was procured from Khari Baoli, Delhi and it was authenticated as the heartwood of Pterocarpus marsupium Roxb. (Ref. No. NISCAIR/RHMD/Consult/2015/2911/104-3) by Dr. Sunita 
Garg, chief scientist, Raw Material Herbarium and Museum, Delhi (RHMD), CSIR-NISCAIR. The voucher specimen is preserved in the herbarium section of Department of Pharmacognosy, KIET School of Pharmacy, Ghaziabad, Uttar Pradesh, India.

\section{Extraction}

The heartwood ( $3 \mathrm{~kg}$ ) was air dried, crushed to smaller pieces, coarsely powdered and extracted with ethanol by cold maceration for $21 \mathrm{~d}$. The ethanolic extract was filtered, concentrated under reduced pressure and dried on a water bath at a temperature below than $75^{\circ} \mathrm{C}$.

\section{Preparation of slurry}

The dried extract $(55 \mathrm{~g}$ ) was dissolved in minimum amount of methanol to attain the desired consistency. Silica gel for column chromatography (60-120 mesh) was added gradually with constant mixing to obtain a slurry. It was air dried and large lumps if any were broken into a smaller size. The uniform particle size of the slurry was obtained by passing it through sieve (\# 8).

\section{Isolation of phytoconstituents}

The dried slurry was chromatographed over silica gel column $(1.6 \mathrm{~m}$ x $16 \mathrm{~mm}$ x $2 \mathrm{~mm}$ ) packed in petroleum ether. The column was eluted successively in increasing order of polarity in various combinations with petroleum ether $\left(60-80{ }^{\circ} \mathrm{C}\right)$, chloroform in petroleum ether $(0.5 \%, 1 \%, 2 \%, 5 \%, 10 \%, 15 \%, 20 \%, 25 \%, 30 \%, 35 \%, 40 \%, 45 \%$, $50 \%, 55 \%, 60 \%, 65 \%, 70 \%, 75 \%, 80 \%, 85 \%, 90 \%, 95 \%$ ) chloroform $(100 \%)$, and methanol in chloroform $(0.1 \%, 0.2 \%, 0.3 \%, 0.4 \%$, $0.5 \%)$. The fractions were collected separately and matched by TLC to check homogeneity. Similar fractions having the same $\mathrm{R}_{\mathrm{f}}$ (retention factor) values were combined and crystallized. The isolated compounds were recrystallized to get pure compounds.

\section{Pass prediction}

In this molecular modelling study, structures were generated with the aid of Chem3D Ultra-9.00 and HyperChem-v.6.02, Wolfram Research Mathematics 6.0 software. Lone pairs of electrons and hydrogen atoms were added where appropriated. The equilibrium geometries of compounds were located using $\mathrm{MM}+($ for Hyper Chem) and MM2 (for Chem3D) functional set. In the next step, RHF calculation (semiempirical AM1 method, the self-consistent field of Hartree-Fock) were performed and bond length, angles, torsion angles and partial charges have been calculated. Calculations were performed on a Intel (R) Core2 (TM) CPU 6600 @ 2.4 GHz Pentium IV computer with 2 GB RAM.

\section{RESULTS}

Phytochemical investigation of the ethanolic extract (dark reddish brown mass, $210.58 \mathrm{~g} \mathrm{(7.02 \% ))} \mathrm{of} \mathrm{the} \mathrm{heartwood} \mathrm{of} \mathrm{Pterocarpus}$ marsupium led to isolate two fatty esters and a phenolic compound characterized as $n$-octanyl $n$-octadeca-9,12-dienoate (n-octanyl linoleiate, 1), $n$-dodecanyl $n$-octadeca-9,12-dienoate ( $n$-dodecanyl linoleiate, 2) and 2, 3-dioxymethylene phenol (3). These phytoconstituents are reported for first time in the plant. The results of the isolation are compiled in table 1.

\section{n-Octanyl linoleiate (1)}

Elution of the column with petroleum ether afforded a dark reddish brown semi-solid mass of 1 , yield $73 \mathrm{mg}(0.13 \%)$; $\mathrm{R}_{\mathrm{f}}$ : 0.90 (chloroform: methanol, 9.5: 0.5), $\mathrm{mp} 74-75^{\circ} \mathrm{C}$; IR $v_{\max }(\mathrm{KBr}): 2927$, $2851,1731,1635,1463,1378,1268,1248,1186,1081,967,719 \mathrm{~cm}^{-}$ 1. ${ }^{1} \mathrm{H}$ NMR $\left(\mathrm{CDCl}_{3}\right): \delta 5.34(1 \mathrm{H}, \mathrm{m}, \mathrm{H}-12), 5.01(1 \mathrm{H}, \mathrm{m}, \mathrm{H}-9), 4.94(1 \mathrm{H}$, $\mathrm{m}, \mathrm{H}-9), 4.90(1 \mathrm{H}, \mathrm{m}, \mathrm{H}-13), 4.09\left(2 \mathrm{H}, \mathrm{d}, \mathrm{J}=6.6 \mathrm{~Hz}, \mathrm{H}_{2}-1^{\prime}\right), 2.78(2 \mathrm{H}, \mathrm{m}$, $\left.\mathrm{H}_{2}-11\right), 2.28\left(2 \mathrm{H}, \mathrm{t}, \mathrm{J}=7.2 \mathrm{~Hz}, \mathrm{H}_{2}-2\right), 2.04\left(2 \mathrm{H}, \mathrm{m}, \mathrm{H}_{2}-8\right), 1.99(2 \mathrm{H}, \mathrm{m}$, $\left.\mathrm{H}_{2}-14\right), 1.56\left(2 \mathrm{H}, \mathrm{m}, \mathrm{CH}_{2}\right), 1.32\left(2 \mathrm{H}, \mathrm{m}, \mathrm{CH}_{2}\right), 1.28\left(6 \mathrm{H}, \mathrm{brs}, 3 \mathrm{x} \mathrm{CH}_{2}\right)$, $1.25\left(16 \mathrm{H}\right.$, brs, $\left.8 \times \mathrm{CH}_{2}\right), 0.89(3 \mathrm{H}, \mathrm{t}, \mathrm{J}=6.5 \mathrm{~Hz}, \mathrm{Me}-18), 0.85(3 \mathrm{H}, \mathrm{t}$, $\left.\mathrm{J}=6.3 \mathrm{~Hz}, \mathrm{Me}-8^{\prime}\right) .{ }^{13} \mathrm{C}$ NMR $\left(\mathrm{CDCl}_{3}\right): \delta 170.63(\mathrm{C}-1), 45.33(\mathrm{C}-2), 30.39$ (C-3), 32.26 (C-4), 29.92 (C-5), 29.55 (C-6), 29.85 (C-7), 32.48 (C-8), 124.20 (C-9), 130.56 (C-10), 37.32 (C-11), 147.30 (C-12), 129.95 (C13), 31.64 (C-14), 29.73 (C-15), 29.67 (C-16), 29.61 (C-17), 14.16 (C18), 63.89 (C-1'), 29.59 (C-2'), 29.38 (C-3'), 29.17 (C-4'), 28.19 (C5'), 27.51 (C-6'), 22.91 (C-7'), 19.94 (C-8'). ESI MS m/z (rel. int.): 392 $[\mathrm{M}]^{+}\left(\mathrm{C}_{26} \mathrm{H}_{48} \mathrm{O}_{2}\right)(25.2), 279$ (41.3), 113 (7.5).

\section{n-Dodecanyl linoleiate (2)}

Elution of the column with petroleum ether-chloroform (9:1) gave a grayish yellow solid mass of 2 , yield $67 \mathrm{mg}(0.12 \%) ; \mathrm{R}_{\mathrm{f}} 0.725$ (chloroform-methanol, 9.5: 0.5), mp 101-102 ${ }^{\circ} \mathrm{C}$; IR $\mathrm{U}_{\max }(\mathrm{KBr}): 2918$, $2849,1740,1620,1442,1376,1238,1101,1081,947,795,729 \mathrm{~cm}$ ${ }^{1} .{ }^{1} \mathrm{H}$ NMR (MeOD): $\delta 5.33(1 \mathrm{H}, \mathrm{m}, \mathrm{H}-12), 4.94(1 \mathrm{H}, \mathrm{m}, \mathrm{H}-10), 4.88$ (2H, m, H-9, H-13), 4.06 (2H, t, J=6.6 Hz, H2-1'), 2.77 (2H, m, $\mathrm{H}_{2}-11$ ), $2.04\left(2 \mathrm{H}, \mathrm{t}, \mathrm{J}=7.2 \mathrm{~Hz}, \mathrm{H}_{2}-2\right), 1.99\left(2 \mathrm{H}, \mathrm{m}, \mathrm{H}_{2}-14\right), 1.83\left(2 \mathrm{H}, \mathrm{m}, \mathrm{H}_{2}-8\right)$, $1.55\left(2 \mathrm{H}, \mathrm{m}, \mathrm{CH}_{2}\right), 1.33\left(2 \mathrm{H}, \mathrm{m}, \mathrm{CH}_{2}\right), 1.30\left(2 \mathrm{H}, \mathrm{m}, \mathrm{CH}_{2}\right), 1.28(30 \mathrm{H}$, brs, $15 \mathrm{x} \mathrm{CH}_{2}$ ), 0.90 (3H, t, J=6.6 Hz, Me-18), $0.87(3 \mathrm{H}, \mathrm{t}, \mathrm{J}=6.4 \mathrm{~Hz}, \mathrm{Me}-$ $12^{\prime}$ ). ${ }^{13} \mathrm{C}$ NMR (MeOD): $\delta 172.31$ (C-1), 40.37 (C-2), 30.89 (C-3), 30.76 (C-4), 30.72 (C-5), 30.66 (C-6), 30.63 (C-7), 33.22 (C-8), 126.21 (C9), 131.54 (C-10), 41.16 (C-11), 142.25 (C-12), 116.15 (C-13), 30.93 (C-14), 30.66 (C-15), 30.63 (C-16), 30.92 (C-17), 14.26 (C-18), 68.45 (C-1'), 30.58(C-2'), 30.55 (C-3'), 30.49 (C-4'), 30.46 (C-5'), 30.42 (C6'), 30.39 (C-7'), 30.37 (C-8'), $30.94\left(\mathrm{C}-9^{\prime}\right), 27.33$ (C-10'), 23.89 (C$\left.11^{\prime}\right), 21.39$ (C-12'). ESI MS m/z (rel. int.): $448[\mathrm{M}]^{+}\left(\mathrm{C}_{30} \mathrm{H}_{56} \mathrm{O}_{2}\right)$ (1.1), $279(39.6)$

\section{2, 3-Dioxymethylene phenol (3)}

Elution of column with chloroform-methanol (99.8: 0.2) yielded a reddish gray solid mass of $3,105 \mathrm{mg}\left(0.19 \%\right.$ yield); $\mathrm{R}_{\mathrm{f}} 0.68$ (chloroform), mp 90-91 ${ }^{\circ} \mathrm{C}$; IR $\mathrm{v}_{\max }(\mathrm{KBr}): 3365,2917,2885,1604$, $1529,1485,1382,1297,1168,1144,1084,964,844 \mathrm{~cm}^{-1} .{ }^{1} \mathrm{H}$ NMR (MeOD): $\delta 6.95(1 \mathrm{H}, \mathrm{m}, \mathrm{H}-6), 6.29(1 \mathrm{H}, \mathrm{m}, \mathrm{H}-4), 6.27(1 \mathrm{H}, \mathrm{m}, \mathrm{H}-5)$, $4.89\left(2 \mathrm{H}, \mathrm{brs},-\mathrm{OCH}_{2}-\mathrm{O}\right) .{ }^{13} \mathrm{C}$ NMR $\left(\mathrm{CDCl}_{3}\right): \delta 159.52(\mathrm{C}-1), 131.04(\mathrm{C}-$ 2), 108.29 (C-3), 107.47 (C-4), 107.88 (C-5), 108.29 (C-6), 103.11 ($\mathrm{OCH}_{2}$-O-). ESI MS m/z (rel. int.): $138[\mathrm{M}]^{+}\left(\mathrm{C}_{7} \mathrm{H}_{6} \mathrm{O}_{3}\right)(1.2)$.

Table 1: Chemical constituents isolated from Pterocarpus marsupium Roxb.

\begin{tabular}{|c|c|c|c|c|c|c|c|c|}
\hline $\begin{array}{l}\text { Comp- } \\
\text { ound }\end{array}$ & $\begin{array}{l}\text { Column } \\
\text { eluant }\end{array}$ & $\begin{array}{l}R_{\mathrm{f}} \text { value mobile } \\
\text { phase }\end{array}$ & $\begin{array}{l}\text { Yield } \\
(\% w / w)\end{array}$ & $\begin{array}{l}\text { Physical } \\
\text { State }\end{array}$ & Colour & $\begin{array}{l}\text { M. p. } \\
{ }^{\circ} \mathbf{C}\end{array}$ & $\begin{array}{l}\text { Mol. wt. } \\
\text { [Mol. for.] }\end{array}$ & Nomenclature \\
\hline (1) & $\mathrm{P}$ & $\begin{array}{l}0.90 \\
C: M(9.5: 0.5)\end{array}$ & $\begin{array}{l}73 \mathrm{mg} \\
(0.13 \%)\end{array}$ & $\begin{array}{l}\text { Semi } \\
\text { solid }\end{array}$ & $\begin{array}{l}\text { Dark reddish } \\
\text { brown }\end{array}$ & $74-75$ & $\begin{array}{l}392 \\
\mathrm{C}_{26} \mathrm{H}_{48} \mathrm{O}_{2}\end{array}$ & $\begin{array}{l}n \text {-octanyl } n \text {-octadeca- } \\
9,12 \text {-dienoate }\end{array}$ \\
\hline (2) & $\begin{array}{l}10 \% \\
C \text { in } P\end{array}$ & $\begin{array}{l}0.725 \\
C: M(9.5: 0.5)\end{array}$ & $\begin{array}{l}67 \mathrm{mg} \\
(0.12 \%)\end{array}$ & solid & Grayish yellow & $\begin{array}{l}101- \\
102\end{array}$ & $\begin{array}{l}448 \\
\mathrm{C}_{30} \mathrm{H}_{56} \mathrm{O}_{2}\end{array}$ & $\begin{array}{l}n \text {-dodecanyl } \\
n \text {-octadeca- } \\
9,12 \text {-dien-oate }\end{array}$ \\
\hline (3) & $\begin{array}{l}0.2 \% \\
M \text { in } C\end{array}$ & $\begin{array}{l}0.68 \\
\text { C: } M(8: 2)\end{array}$ & $\begin{array}{l}105 \mathrm{mg} \\
(0.19 \%)\end{array}$ & solid & Reddish gray & $90-91$ & $\begin{array}{l}138 \\
\mathrm{C}_{7} \mathrm{H}_{6} \mathrm{O}_{3}\end{array}$ & $\begin{array}{l}\text { 2, 3-dioxymethylene } \\
\text { phenol }\end{array}$ \\
\hline
\end{tabular}

P-Petroleum ether $\left(60-80^{\circ} \mathrm{C}\right), \mathrm{C}-$ Chloroform; M-Methanol

The results of PASS prediction which revealed the biological activity spectra of the isolated compound (1), (2) and (3) are depicted in table 2, 3 and 4 respectively.

The first column in these tables show the percentage activity and the second show the percentage inactivity for the activity mentioned in column 3.

\section{DISCUSSION}

The compound 1 was obtained as a dark reddish brown semi-solid substance from petroleum ether eluants. Its IR spectrum exhibited characteristic absorption bands for ester group $\left(1731 \mathrm{~cm}^{-1}\right)$, unsaturation (1635 $\left.\mathrm{cm}^{-1}\right)$ and long aliphatic chain $\left(719 \mathrm{~cm}^{-1}\right)$. The mass spectrum of 1 had a molecular ion peak at $m / z 392$ consistent 
with the molecular formula an aliphatic ester, $\mathrm{C}_{26} \mathrm{H}_{48} \mathrm{O}_{2}$. The prominent fragments generated at $m / z \quad 279 \quad\left[\mathrm{CH}_{3} \quad\left(\mathrm{CH}_{2}\right)_{3}\right.$ $\left.\left(\mathrm{CH}_{2} \mathrm{CH}=\mathrm{CH}\right)_{2}\left(\mathrm{CH}_{2}\right)_{7} \mathrm{COO}\right]^{+}$and $113\left[\left(\mathrm{CH}_{2}\right)_{7} \mathrm{CH}_{3}\right]^{+}$suggested that $n$ octanol was esterified with linoleic acid. The ${ }^{1} \mathrm{H}$ NMR spectrum of 1 showed four one-proton multiplets at $\delta 5.34,5.01,4.94$ and 4.90 attributed to vinylic $\mathrm{H}-12, \mathrm{H}-10, \mathrm{H}-9$ and $\mathrm{H}-13$ protons, respectively. A two-proton doublet at $\delta 4.09(\mathrm{~J}=6.6 \mathrm{~Hz})$ and a two-proton triplet at $\delta 2.28(\mathrm{~J}=7.2 \mathrm{~Hz})$ were ascribed to oxygenated methylene $\mathrm{H}_{2}-1^{\prime}$ and methylene $\mathrm{H}_{2}-2$ adjacent to the ester group, respectively. Five twoproton multiplets between $\delta$ 2.78-1.32 and two broad singlets at $\delta$ $1.28(6 \mathrm{H})$ and $1.25(16 \mathrm{H})$ were associated with the remaining methylene protons.

Two-three proton triplets at $\delta 0.89(\mathrm{~J}=6.5 \mathrm{~Hz})$ and $\delta 0.85(\mathrm{~J}=6.3 \mathrm{~Hz})$ were assigned to the terminal primary Me-18 and Me- $8^{\prime}$ protons, respectively. The ${ }^{13} \mathrm{C}$ NMR spectrum of 1 exhibited the presence of ester carbon at $\delta 170.63(\mathrm{C}-1)$, vinylic carbons between $\delta 147.30$ 124.20 and methyl carbons at $\delta 14.16(\mathrm{C}-18)$ and $19.94\left(\mathrm{C}^{\prime} \mathbf{8}^{\prime}\right)$. On the basis of the spectral data analysis, the structure of 1 has been established as $n$-octanyl $n$-octadeca-9, 12 -dienoate. This phytoconstituent is first time reported in P. marsupium.

$$
\begin{aligned}
& \begin{array}{lllllllll}
18 & 13 & 12 & 11 & 10 & 9 & 1 & 1^{\prime} & 8^{\prime}
\end{array} \\
& \mathrm{CH}_{3}\left(\mathrm{CH}_{2}\right)_{4} \mathrm{CH}=\mathrm{CHCH}_{2} \mathrm{CH}=\mathrm{CH}\left(\mathrm{CH}_{2}\right)_{7} \mathrm{COOCH}_{2}\left(\mathrm{CH}_{2}\right)_{6} \mathrm{CH}_{3}
\end{aligned}
$$

\section{n-Octanyl $n$-octadeca-9,12-dienoate (1)}

The compound $\mathbf{2}$ was a greyish yellow solid mass isolated from petroleum ether-chloroform (9:1) eluants. Its IR spectrum showed distinctive absorption bands for ester group $\left(1740 \mathrm{~cm}^{-1}\right)$, unsaturation (1620 $\left.\mathrm{cm}^{-1}\right)$ and long aliphatic chain $\left(729 \mathrm{~cm}^{-1}\right)$. The mass spectrum of 2 exhibited a molecular ion peak at $m / z 448$ corresponding to the molecular formula an aliphatic ester, $\mathrm{C}_{30} \mathrm{H}_{56}$ $\mathrm{O}_{2}$. The prominent fragment generated at $\mathrm{m} / \mathrm{z} 279\left[\mathrm{CH}_{3}\left(\mathrm{CH}_{2}\right)_{3}\right.$ $\left.\left(\mathrm{CH}_{2} \mathrm{CH}=\mathrm{CH}\right)_{2}\left(\mathrm{CH}_{2}\right)_{7} \mathrm{COO}\right]^{+}$indicated that linoleic acid was esterified with an aliphatic alcohol. The ${ }^{1} \mathrm{H}$ NMR spectrum of $\mathbf{2}$ displayed two one-proton multiplets at $\delta 5.33$ and 4.94 and a two-proton multiplet 4.88 ascribed to vinylic $\mathrm{H}-12, \mathrm{H}-10, \mathrm{H}-9$ and $\mathrm{H}-13$ protons, respectively. Two two-proton triplets at $\delta 4.06(\mathrm{~J}=6.6 \mathrm{~Hz}$ ) and 2.04 $\left(\mathrm{J}=7.2 \mathrm{~Hz}\right.$ ) were ascribed to oxygenated methylene $\mathrm{H}_{2}-1^{\prime}$ and methylene $\mathrm{H}_{2}-2$ adjacent to the ester group, respectively.

Six two-proton multiplets between $\delta$ 2.77-1.30 and a broad singlet at $\delta 1.28(30 \mathrm{H})$ were associated with the remaining methylene protons. Two three-proton triplets at $\delta 0.90(\mathrm{~J}=6.6 \mathrm{~Hz})$ and $\delta 0.87$ $(\mathrm{J}=6.4 \mathrm{~Hz})$ were assigned correspondingly to the terminal primary Me-18 and Me-12' protons. The ${ }^{13} \mathrm{C}$ NMR spectrum of 2 showed the presence of ester carbon at $\delta 172.31(\mathrm{C}-1)$, vinylic carbons between $\delta 142.25-116.15$ and methyl carbons at $\delta 14.26(\mathrm{C}-18)$ and 21.39 (C$12^{\prime}$ ). On the basis of these evidence, the structure of 2 has been elucidated as $n$-dodecanyl $n$-octadeca-9, 12-dienoate. This phytoconstituent is first time reported in P. marsupium.

$$
\stackrel{18}{\mathrm{CH}_{3}}\left(\mathrm{CH}_{2}\right)_{4} \stackrel{13}{\mathrm{CH}} \stackrel{12}{\mathrm{C}} \stackrel{11}{\mathrm{C}} \stackrel{10}{\mathrm{HCH}} \mathrm{H}_{2} \mathrm{CH}=\stackrel{9}{\mathrm{C}} \mathrm{H}\left(\mathrm{CH}_{2}\right)_{7} \stackrel{1}{\mathrm{C}} \stackrel{1^{\prime}}{\mathrm{O}} \mathrm{CH}_{2}\left(\mathrm{CH}_{2}\right)_{10} \stackrel{12}{\mathrm{C}} \mathrm{H}_{3}^{\prime}
$$

n-Dodecanyl $n$-octadeca-9,12-dienoate (2)

The compound $\mathbf{3}$ was obtained as a reddish grey solid substance from chloroform: methanol (99.8: 0.2) eluants. It responded positive tests for phenol and had a molecular ion peak at $m / z 138$ in its mass spectrum corresponding to the molecular formula of a phenolic compound, $\mathrm{C}_{7} \mathrm{H}_{6} \mathrm{O}_{3}$. Its IR spectrum exhibited distinct absorption bands for hydroxyl group (3365 $\mathrm{cm}^{-1}$ ) and aromatic ring (1604, $\left.1529,1084 \mathrm{~cm}^{-1}\right)$. The ${ }^{1} \mathrm{H}$ NMR spectrum of $\mathbf{3}$ showed three oneproton multiplets at $\delta 6.95,6.29$ and 6.27 due to aromatic H-6, H-4 and $\mathrm{H}-5$ protons, respectively.

A two-proton broad singlet at $\delta 4.89$ was attributed to di-oxygenated methylene,-0- $\mathrm{CH}_{2}-\mathrm{O}-$, protons. Its ${ }^{13} \mathrm{C}$ NMR spectrum displayed signals for aromatic carbons from $\delta 159.52$ to 107.47 , and dioxygenated methylene carbon at $\delta 103.11$. These spectral data led to formulate the structure of $\mathbf{3}$ as 2, 3-dioxymethylene phenol. This phytoconstituent is first time reported in P. marsupium.<smiles>Oc1cccc2c1OCO2</smiles>

\section{2, 3-Dioxymethylene phenol (3)}

The PASS computer program, which is able to simultaneously predict more than one thousand biological and toxicological activities from only the structural formulas of the chemicals, was used to predict the biological activity profile of all the three new compounds isolated from Pterocarpus marsupium. Various novel pharmaceuticals have been discovered using PASS prediction. This application is a wonderful gift of technology to the mankind because it helps to discover new, potent and safe medicinal agents. It also provides an excellent opportunity for the multidisciplinary professionals (e. g. pharma and computational experts) to work together on a single platform and serve the society.

The PASS prediction profiles of the isolated compounds in the current research investigation have contributed towards the incredibility of this piece of work. n-octanyl linoleiate (1) showed the highest probability to be active as All-trans-retinyl-palmitate hydrolase inhibitor, anti eczematic, lipid metabolism regulator, CYP2J substrate, CYP2J2 substrate, phosphatidylcholine-retinol Oacyltransferase inhibitor, lipoprotein lipase inhibitor, phosphatidyl glycerophosphatase inhibitor, muco-membranous protector and alkenyl glycerophosphocholine hydrolase inhibitor. n-dodecanyl linoleiate (2) also exhibited a similar biological activity spectrum.

Table 2: Biological activity of n-octanyl linoleiate (1)

\begin{tabular}{lll}
\hline Percentage activity & Percentage inactivity & Name of activity \\
\hline 96.4 & 0.1 & All-trans-retinyl-palmitate hydrolase inhibitor \\
95.6 & 0.2 & Antieczematic \\
92.9 & 0.3 & Lipid metabolism regulator \\
92.6 & 0.3 & CYP2J substrate \\
92.0 & 0.3 & CYP2J2 substrate \\
91.5 & 0.2 & Phosphatidylcholine-retinol O-acyltransferase inhibitor \\
91.1 & 0.3 & Lipoprotein lipase inhibitor \\
90.2 & 0.2 & Phosphatidylglycerophosphatase inhibitor \\
90.4 & 0.5 & Mucomembranous protector \\
89.9 & 0.5 & Alkenylglycerophosphocholine hydrolase inhibitor \\
89.0 & 0.4 & Alkylacetylglycerophosphatase inhibitor \\
88.6 & 0.3 & Cutinase inhibitor \\
87.9 & 0.5 & Acylcarnitine hydrolase inhibitor \\
86.9 & 0.4 & Cholesterol antagonist \\
86.7 & 0.9 & Polyporopepsin inhibitor \\
86.6 & 0.9 & Saccharopepsin inhibitor \\
86.0 & 0.3 & Macrophage colony stimulating factor agonist \\
85.3 & 0.4 & Dextranase inhibitor \\
\hline
\end{tabular}




\begin{tabular}{|c|c|c|}
\hline 84.7 & 0.5 & Fucosterol-epoxide lyase inhibitor \\
\hline 84.1 & 0.3 & Preneoplastic conditions treatment \\
\hline 83.9 & 0.4 & IgA-specific serine endopeptidase inhibitor \\
\hline 83.7 & 0.4 & Antihypercholesterolemic \\
\hline 83.3 & 0.4 & Antisecretoric \\
\hline 83.0 & 0.2 & Angiogenesis stimulant \\
\hline 82.3 & 0.5 & Linoleate diol synthase inhibitor \\
\hline 82.1 & 0.3 & Phosphatidate phosphatase inhibitor \\
\hline 81.8 & 0.4 & Poly(alpha-L-guluronate) lyase inhibitor \\
\hline 81.0 & 0.2 & Protein-tyrosine sulfotransferase inhibitor \\
\hline 80.6 & 0.3 & Poly(beta-D-mannuronate) lyase inhibitor \\
\hline 82.5 & 2.5 & Ubiquinol-cytochrome-c reductase inhibitor \\
\hline 79.9 & 0.1 & Thromboxane synthase stimulant \\
\hline 78.0 & 0.4 & Leukopoiesis stimulant \\
\hline 77.9 & 0.5 & IgA-specific metalloendopeptidase inhibitor \\
\hline 76.0 & 0.3 & Ethanolamine-phosphate cytidylyltransferase inhibitor \\
\hline 75.8 & 0.3 & Gastrin inhibitor \\
\hline 75.7 & 0.2 & Pediculicide \\
\hline 76.2 & 0.9 & Oxidoreductase inhibitor \\
\hline 75.3 & 0.4 & Lactase inhibitor \\
\hline 74.5 & 0.1 & Cyclooxygenase 1 substrate \\
\hline 73.5 & 1.1 & Membrane integrity antagonist \\
\hline 72.5 & 0.4 & Leukotriene-B4 20-monooxygenase inhibitor \\
\hline 72.0 & 0.7 & Levanase inhibitor \\
\hline 71.5 & 0.8 & CYP3A1 substrate \\
\hline 72.4 & 2.4 & Pro-opiomelanocortin converting enzyme inhibitor \\
\hline 70.3 & 0.5 & Cytoprotectant \\
\hline
\end{tabular}

Table 3: Biological activity of n-dodecanyl linoleiate (2)

\begin{tabular}{|c|c|c|}
\hline Percentage activity & Percentage inactivity & Name of activity \\
\hline 96.4 & 0.1 & All-trans-retinyl-palmitate hydrolase inhibitor \\
\hline 95.6 & 0.2 & Antieczematic \\
\hline 92.9 & 0.3 & Lipid metabolism regulator \\
\hline 92.0 & 0.3 & CYP2J2 substrate \\
\hline 91.5 & 0.3 & GST A substrate \\
\hline 90.2 & 0.2 & Phosphatidylglycerophosphatase inhibitor \\
\hline 90.4 & 0.5 & Mucomembranous protector \\
\hline 89.0 & 0.4 & Alkylacetylglycerophosphatase inhibitor \\
\hline 88.4 & 0.1 & Alcohol 0-acetyltransferase inhibitor \\
\hline 87.2 & 0.1 & Endocannabinoid uptake inhibitor \\
\hline 86.6 & 0.9 & Saccharopepsin inhibitor \\
\hline 86.0 & 0.3 & Macrophage colony stimulating factor agonist \\
\hline 85.4 & 0.5 & Pullulanase inhibitor \\
\hline 84.7 & 0.5 & Fucosterol-epoxide lyase inhibitor \\
\hline 84.1 & 0.3 & Preneoplastic conditions treatment \\
\hline 83.7 & 0.5 & Exoribonuclease II inhibitor \\
\hline 83.0 & 0.2 & Angiogenesis stimulant \\
\hline 82.3 & 0.5 & Linoleate diol synthase inhibitor \\
\hline 81.8 & 0.2 & Phosphatidylinositol diacylglycerol-lyase inhibitor \\
\hline 81.0 & 0.2 & Protein-tyrosine sulfotransferase inhibitor \\
\hline 80.7 & 0.4 & CYP2E1 inhibitor \\
\hline 79.9 & 0.1 & Thromboxane synthase stimulant \\
\hline 78.8 & 0.4 & Ecdysone 20-monooxygenase inhibitor \\
\hline 78.0 & 0.4 & Leukopoiesis stimulant \\
\hline 77.9 & 0.5 & IgA-specific metalloendopeptidase inhibitor \\
\hline 76.2 & 0.2 & Vanilloid 1 agonist \\
\hline 76.0 & 0.5 & Sarcosine oxidase inhibitor \\
\hline 75.3 & 0.4 & Lactase inhibitor \\
\hline 74.8 & 0.4 & Anthranilate-CoA ligase inhibitor \\
\hline 73.2 & 0.4 & Cyclomaltodextrinase inhibitor \\
\hline 73.3 & 0.5 & Antimutagenic \\
\hline 73.5 & 1.1 & Membrane integrity antagonist \\
\hline 72.5 & 0.4 & Leukotriene-B4 20-monooxygenase inhibitor \\
\hline 73.4 & 1.5 & Arginine 2-monooxygenase inhibitor \\
\hline 72.3 & 0.5 & Alkenylglycerophosphoethanolamine hydrolase inhibitor \\
\hline 72.0 & 0.7 & Levanase inhibitor \\
\hline 71.4 & 0.3 & Sclerosant \\
\hline 71.5 & 0.8 & CYP3A1 substrate \\
\hline 70.6 & 0.3 & CYP4A substrate \\
\hline 70.3 & 0.5 & Cytoprotectant \\
\hline 70.4 & 0.8 & HMOX1 expression enhancer \\
\hline
\end{tabular}


This also proves the fact that the structure of the compound is related to its activity and also that compounds with similar chemical structure exhibit similarity in their activity profiles also. 2, 3dioxymethylene phenol (3) was found to be active as membrane integrity agonist, aspulvinone dimethylallyl transferases inhibitor, carminative, neurotransmitter uptake inhibitor, a dehydro-Lgulonate decarboxylase inhibitor, MAP kinase stimulant, ubiquinolcytochrome-c reductase inhibitor, JAK2 expression inhibitor, NADPH peroxidase inhibitor, glutathione thioesterase inhibitor, GABA aminotransferase inhibitor and antiseptic.

Table 4: Biological activity of 2, 3-Dioxymethylene phenol (3)

\begin{tabular}{|c|c|c|}
\hline Percentage activity & Percentage inactivity & Name of activity \\
\hline 95.2 & 0.3 & Membrane Integrity Agonist \\
\hline 90.6 & 0.7 & Aspulvinone dimethylallyl transferases inhibitor \\
\hline 89.9 & 0.2 & Carminative \\
\hline 87.8 & 0.2 & Neurotransmitter uptake inhibitor \\
\hline 82.7 & 0.7 & Dehydro-L-gulonate decarboxylase inhibitor \\
\hline 81.3 & 0.3 & MAP kinase stimulant \\
\hline 81.8 & 0.27 & Ubiquinol-cytochrome-c reductase inhibitor \\
\hline 79.8 & 0.8 & JAK2 expression inhibitor \\
\hline 79.9 & 1.2 & NADPH peroxidase inhibitor \\
\hline 79.3 & 0.8 & Glutathione thiolesterase inhibitor \\
\hline 78.5 & 0.3 & GABA aminotransferase inhibitor \\
\hline 77.9 & 0.4 & Antiseptic \\
\hline 80.7 & 3.5 & CYP2C12 substrate \\
\hline 79.3 & 2.1 & Chlordecone reductase inhibitor \\
\hline 77.3 & 0.7 & Antidyskinetic \\
\hline 77.8 & 1.4 & Feruloyl esterase inhibitor \\
\hline 76.3 & 0.8 & Caspase 3 stimulant \\
\hline 77.8 & 2.3 & Antiseborrheic \\
\hline 75.6 & 0.4 & MMP9 expression inhibitor \\
\hline 75.8 & 1.0 & Alkane 1-monooxygenase inhibitor \\
\hline 76.3 & 2.2 & Sugar-phosphatase inhibitor \\
\hline 76.1 & 2.3 & Methylenetetrahydrofolate reductase (NADPH) inhibitor \\
\hline 77.1 & 3.3 & Testosterone 17 beta-dehydrogenase (NADP+) inhibitor \\
\hline 74.2 & 1.4 & Glucan endo-1,6-beta-glucosidase inhibitor \\
\hline 74.6 & 2.2 & Nicotinic alpha6beta3beta4alpha5 receptor antagonist \\
\hline 74.2 & 2.6 & Alkenylglycerophosphocholine hydrolase inhibitor \\
\hline 73.4 & 2.0 & Glucose oxidase inhibitor \\
\hline 72.3 & 1.4 & NADPH-cytochrome-c2 reductase inhibitor \\
\hline 71.4 & 0.5 & Ovulation inhibitor \\
\hline 71.5 & 0.6 & Anesthetic general \\
\hline 74.0 & 3.4 & Acrocylindropepsin inhibitor \\
\hline 74.0 & 3.4 & Chymosin inhibitor \\
\hline 74.0 & 3.4 & Saccharopepsin inhibitor \\
\hline 71.9 & 1.7 & Ribulose-phosphate 3-epimerase inhibitor \\
\hline 70.9 & 0.8 & Ecdysone 20-monooxygenase inhibitor \\
\hline 70.4 & 1.0 & Thioredoxin inhibitor \\
\hline 70.4 & 1.8 & Complement factor D inhibitor \\
\hline 70.7 & 2.6 & Glutamyl endopeptidase II inhibitor \\
\hline 70.0 & 1.6 & Arylacetonitrilase inhibitor \\
\hline 70.7 & 2.6 & Glutamyl endopeptidase II inhibitor \\
\hline
\end{tabular}

\section{CONCLUSION}

Currently, PASS web-service is being utilised by more than 8700 registered users from more than 7o countries. Predictions for more than 250,000 organic compounds have been obtained from this computer program. More than 4,000 pharmacological effects, specific toxicities, mode of action, effect on gene expression, the interaction of metabolic enzymes, etc. have been predicted so far.

The isolated phytoconstituents in the current investigation are being reported for the first time so these can be utilised as fingerprinting markers for the various chromatographic techniques for establishing the identity, quality and purity of the drug. The in silico profiling of these phytoconstituents shall be more beneficial than the animal studies because of the significant variation in the genetic pattern of the humans and the rodents. The results of this study shall be very helpful for the upcoming research investigations to establish these new compounds as the pharmacologically active moieties.

\section{ACKNOWLEDGEMENT}

The authors are thankful to the KIET central library and NISCAIR library, New Delhi for providing the literature and the head, SAIF
Division, CDRI, Lucknow for recording spectral data of the compounds.

\section{CONFLICTS OF INTERESTS}

There are no conflicts of interest.

\section{REFERENCES}

1. Sharma V, Chaudhary U. Pharmacognostical and phytochemical screening of Helicteres isora roots. Asian J Pharm Clin Res 2016;9:96-101.

2. Kripa KG, Sangeetha R, Chamundeeswari D. Pharmacognostical and physicochemical evaluation of the plant Leucas Aspera. Asian J Pharm Clin Res 2016;9:263-8.

3. Saldanha CJ. Flora of Karnataka. Vol. I. Oxford: IBH Publishing; 1984. p. 21.

4. Matthew KM. The Flora of Tamil Nadu Carnatic. St. Josephs College: Tiruchirapalli, India; 1983.

5. Warrier PK. Indian medicinal plants: a compendium of 500 species. Vol. 3. Universities Press; 1995. p. 280.

6. Pullaiah T. Medicinal plants of Andhra Pradesh. New Delhi, India: Regency Publications; 1999. p. 63. 
7. Devgun M, Nanda A, Ansari SH. Pterocarpus marsupium Roxb.-A comprehensive review. Pharmacogn Rev 2009;3:359-63.

8. Katiyar D, Singh V, Ali M. Phytochemical and pharmacological profile of Pterocarpus marsupium: a review. Pharma Innovation J 2016;5:31-9.

9. Maurya R, Ray AB. Constituents of Pterocarpus marsupium. J Nat Prod 1984;47:179-81.

10. Chakravarthy BK, Gode KD. Isolation of (-) Epicatechin from Pterocarpus marsupium and its pharmacological actions. Planta Med 1985;51:56-9.

11. Tripathi J, Joshi T. Flavonoids from Pterocarpus marsupium. Planta Med 1988;54:371-2.

12. Tripathi J, Joshi T. Phytochemical investigation of roots of Pterocarpus marsupium: Isolation and structural studies of two new flavanone glycosides. Z Naturforsch C 1988;43:184-6.

13. Jahromi MAF, Ray AB. Antihyperlipidemic effect of flavonoids from Pterocarpus marsupium. J Nat Prod 1993;56:989-94.

14. Handa SS, Singh R, Maurya R, Satti NK, Suri KA, Suri OP. Pterocaroside, an isoaurone C-glucoside from Pterocarpus marsupium. Tetrahedron Lett 2000;41:1579-81.
15. Suri KA, Satti NK, Gupta BD, Suri OP. 1-(2', 6' dihydroxyphenyl)-(i-D-glucopyranoside, a novel C-glycoside from Pterocarpus marsupium. Indian J Chem 2003;42B:432-3.

16. Maurya R, Singh R, Deepak M, Handa SS, Yadav PP, Mishra PK. Constituents of Pterocarpus marsupium: a crude ayurvedic drug. Phytochemicals 2004;65:915-20.

17. Lagunin A, Stepanchikova A, Filimonov D, Poroikov, PASS: prediction of activity spectra for biologically active substances. Bioinformatics Applications Note 2000;16:747-8.

18. Porovikov V, Filimonov D, Borodina Yu, Langunin A, Kos A Robustness of biological activity spectra predicting by computer program PASS for non-Congeneric sets of chemical compounds. J Chem Inf Comput Sci 2000;40:1349-55.

\section{How to cite this article}

- Deepti Katiyar, Vijender Singh, Mohammed Ali. Isolation, characterization and prediction of the biological activity of two new fatty esters and a phenol from the heartwood of Pterocarpus marsupium roxb. Int J Pharm Pharm Sci 2017;9(2):117-122. 